\title{
A NONLINEAR TWO-SPECIES OSCILLATORY SYSTEM: BIFURCATION AND STABILITY ANALYSIS
}

\author{
MALAY BANDYOPADHYAY, RAKHI BHATTACHARYA, \\ and C. G. CHAKRABARTI
}

Received 20 January 2002

\begin{abstract}
The present paper dealing with the nonlinear bifurcation analysis of two-species oscillatory system consists of three parts. The first part deals with Hopf-bifurcation and limit cycle analysis of the homogeneous system. The second consists of travelling wave train solution and its linear stability analysis of the system in presence of diffusion. The last deals with an oscillatory chemical system as an illustrative example.
\end{abstract}

2000 Mathematics Subject Classification: 37G15.

1. Introduction. Periodicity is an inherent phenomenon in living systems. From the cell cycle, which governs the rate and timing of mitosis (cell division), to the diurnal cycle that results in sleep-wake, to the ebb and flow of populations in their natural environment, life proceeds in a rhythmic and periodic style [6]. Within the nature, several dynamical systems exhibit a large variety of oscillations. The spring-mass system, electrical circuits, Lotka-Volterra predation model system, and so forth. exhibit several types of periodic behaviour. There are some stable periodic behaviours which are not easily disrupted by a perturbation, deterministic or random. These types of situations lead us to believe that pattern is a ubiquitous part of the process of growth of biochemical and metabolic control systems and of ecological systems.

Reaction-diffusion processes play a significant role in the study of pattern formation in different biological and ecological system [6, 16]. A large class of nonlinear parabolic partial differential equations are referred to as reactiondiffusion equations [8]. The systems governed by this type of equations are known as reaction-diffusion system. For example, if $u_{i}(x, t), i=1,2, \ldots, m$ represents the densities or concentrations of several interacting species or chemicals each of which diffusing with their own diffusion coefficients $D_{i}$ and interacting according to the vector source term $\mathbf{f}$, then the system is governed by [16],

$$
\frac{\partial \mathbf{u}}{\partial t}=\mathbf{f}+D \nabla^{2} \mathbf{u}
$$

where $D$ is a simple diagonal matrix of order $m$ for the case of no cross diffusion. Equation (1.1) is referred to as a reaction-diffusion or an interacting 
population diffusion system [16]. It is believed that rotating and spiral waves are possible solutions of reaction-diffusion equations in appropriate circumstances. Rotating spiral waves have been found by Winfree [21] for the Belousov-Zhabotinskii reaction. Kuramoto and Yamada [15] considered a twospecies reaction-diffusion system exhibiting limit cycle behaviour. Cohen et al. [3] were the first to demonstrate that the rotating spiral wave can be maintained by a reaction-diffusion mechanism alone. They found solutions for the $\lambda-\omega$ system (see [3])

$$
\begin{aligned}
& \frac{\partial u}{\partial t}=\lambda(R) u-\omega(R) v+\nabla^{2} u \\
& \frac{\partial v}{\partial t}=\omega(R) u+\lambda(R) v+\nabla^{2} v
\end{aligned}
$$

where $\lambda, \omega$ are given functions of $R=\left(u^{2}+v^{2}\right)^{1 / 2}$. Stability of travelling waves can often be quite difficult to demonstrate analytically. However, some stability results can be obtained, without long and complicated analysis, in the case of the wave train solutions of the $\lambda-\omega$ system [16]. Feroe [7] investigated the systems for which this stability analysis is possible or not.

The object of the present work is to develop a limit cycle solution of a general nonlinear two-species model system and then to obtain the criteria of the stability. In Section 3, we have tried to find the travelling wave train solution of the above-mentioned problem in presence of diffusive perturbation for both species. We have also performed the linear stability analysis for the travelling wave train solution. As an illustrative example, we have considered a nonlinear reaction-diffusion model equation which governs a certain chemical reaction system introduced by Dreitlein and Somes [4] for which the criterion of linear stability for travelling wave train solution have been tested.

2. A nonlinear system: Hopf-bifurcation and limit cycles. We consider a nonlinear system of two interacting species (ecological or chemical) whose concentrations are denoted by $x_{1}(t)$ and $x_{2}(t)$ and is governed by the system of equations [17]

$$
\begin{aligned}
& \frac{d x_{1}}{d t}=\gamma x_{1}-\omega x_{2}+\left(m x_{1}-n x_{2}\right)\left(x_{1}^{2}+x_{2}^{2}\right), \\
& \frac{d x_{1}}{d t}=\omega x_{1}+\gamma x_{2}+\left(n x_{1}+m x_{2}\right)\left(x_{1}^{2}+x_{2}^{2}\right),
\end{aligned}
$$

where $\gamma$ is a scalar control parameter and $\omega, m, n$ are constants. This highly nonlinear planar model is a generalization of various types of nonlinear differential equations governing a variety of physical and chemical systems [2]. Evidently, $\left(x_{1}^{*}, x_{2}^{*}\right)=(0,0)$ is the fixed point of (2.1) for all values of the control parameter $\gamma$. Let the matrix $\mathbf{A}(\gamma)$ be the linearized matrix of (2.1) about 
the fixed point $\left(x_{1}^{*}, x_{2}^{*}\right)$, that is,

$$
\mathbf{A}(\gamma)=\left[\nabla_{\mathbf{X}} \mathbf{F}(\mathbf{X}, \gamma)\right]_{\left(\mathbf{X}^{*}\right)}=\left(\begin{array}{cc}
\gamma & -\omega \\
\omega & \gamma
\end{array}\right)
$$

where

$$
\begin{aligned}
\mathbf{X} & =\left[x_{1}, x_{2}\right]^{T}, \quad \mathbf{F}[\mathbf{X}, \gamma]=\left[F_{1}, F_{2}\right]^{T}, \\
F_{1} & =\gamma x_{1}-\omega x_{2}+\left(m x_{1}-n x_{2}\right)\left(x_{1}^{2}+x_{2}^{2}\right), \\
F_{2} & =\omega x_{1}+\gamma x_{2}+\left(n x_{1}+m x_{2}\right)\left(x_{1}^{2}+x_{2}^{2}\right) .
\end{aligned}
$$

The eigenvalues of the corresponding Jacobian matrix (2.2) are

$$
\lambda_{1}=\gamma-i \omega, \quad \lambda_{2}=\gamma+i \omega
$$

From these eigenvalues, we note that $(0,0)$ is a nonhyperbolic fixed point $[11,12,18,19]$ of $(2.1)$ when $\gamma=0$. Further at $\left(x_{1}, x_{2}, \gamma\right)=(0,0,0)$,

$$
\frac{d \lambda_{1}}{d \gamma}=1, \quad \frac{d \lambda_{2}}{d \gamma}=1
$$

Hence, all the conditions required for a Hopf-bifurcation are satisfied [9, $16,17]$. Alternatively, the matrix $\mathbf{A}(0)$ has purely imaginary eigenvalues $\pm i \omega$ $(\omega \neq 0)$, that is, the conditions [16]

$$
\operatorname{Tr} \mathbf{A}(0)=0, \quad \operatorname{det} \mathbf{A}(0)=\omega^{2}>0
$$

are satisfied. Also the matrix $\mathbf{B}(\gamma)$ defined by

$$
\mathbf{A}(\gamma)=\mathbf{A}(0)+\gamma \mathbf{B}(\gamma)
$$

is such that $\operatorname{Tr} \mathbf{B}(0)=2 \neq 0$, so there must exist a periodic solution of (2.1) for $\gamma$ in some neighborhood of $\gamma=0$ and $\mathbf{X}$ in some neighborhood of $\mathbf{X}^{*}$ with approximate period $T=2 \pi / \omega$ for small $\gamma$ [16]. We can now apply Hopf-bifurcation and limit cycle theorem [16] to find the periodic solution of the system of (2.1). Following Murray [16] and without going into the details of calculation, we can show that the periodic solution of (2.1) is given by

$$
\mathbf{X}(\gamma, t)=\left(\begin{array}{l}
x_{1} \\
x_{2}
\end{array}\right)=\left[-\frac{\gamma\left(1+\omega^{2}\right)}{2 m}\right]^{1 / 2}\left(\begin{array}{l}
\cos (\omega t+\alpha) \\
\sin (\omega t+\alpha)
\end{array}\right)+o(\gamma),
$$

where $\alpha=\arctan \left(b_{2} / b_{1}\right)$ is some arbitrary phase angle. From (2.8), we see that the amplitude of oscillations depends on the parameters $\gamma, \omega$, and $m$. For the existence of the limit cycle, the amplitude should be positive and this requires $\gamma$ and $m$ to be of opposite sign [1]. Now we consider the stability of the limit cycle (2.8). For this we calculate the Lyapunov-number $\sigma$ for system (2.1). 
Following Perko [18], we can calculate the Lyapunov-number $\sigma$ for system (2.1) about the stationary state $\mathbf{X}^{*}$ as

$$
\sigma=\frac{12 \pi}{\omega} m
$$

If $\sigma \neq 0$, the fixed point $(0,0)$ is a weak focus of multiplicity one. The weak focus will be stable or unstable according to whether $\sigma<0$ or $\sigma>0$, that is, according to whether $\omega m<0$ or $\omega m>0$. Hopf-bifurcation occurs at the critical value $\gamma=0$. If $\sigma<0$ or $\omega m<0$, the Hopf-bifurcation is supercritical and, on the other hand, if $\sigma>0$ or $\omega m>0$, the Hopf-bifurcation is subcritical.

3. Nonlinear reaction-diffusion system: travelling wave trains and linear stability analysis. In this section, we consider the behaviour of the system governed by (2.1) in presence of diffusion. We try to find the wave train solutions for the reaction-diffusion system given by

$$
\begin{aligned}
& \frac{\partial x_{1}}{\partial t}=\gamma x_{1}-\omega x_{2}+\left(m x_{1}-n x_{2}\right)\left(x_{1}^{2}+x_{2}^{2}\right)+\frac{\partial^{2} x_{1}}{\partial x^{2}}, \\
& \frac{\partial x_{1}}{\partial t}=\omega x_{1}+\gamma x_{2}+\left(n x_{1}+m x_{2}\right)\left(x_{1}^{2}+x_{2}^{2}\right)+\frac{\partial^{2} x_{2}}{\partial x^{2}} .
\end{aligned}
$$

For our purpose here, we consider the system with the same rate of diffusion for both species and then it is scaled into a new space variable by using the transformation $x \rightarrow x / \sqrt{D}$, where $D$ is the same rate of diffusion for both species. We have shown in the previous section that $\gamma$ is a bifurcation parameter and when it passes through the value zero, Hopf-bifurcation takes place. We assume the travelling wave train solution of system (3.1) in the form

$$
\mathbf{v}(x, t)=\left(\begin{array}{l}
x_{1}(x, t) \\
x_{2}(x, t)
\end{array}\right)=\mathbf{V}(z), \text { where } z=\sigma t-k x,
$$

with $\sigma$ (>0) being the frequency of the wave train, $k$ the wave number, and $\mathbf{V}$ a periodic function of $z$ with period $2 \pi$. Then the wavelength is $\omega=2 \pi / k$ and the wave propagates with the speed $c=\sigma / k$. Substitution of (3.2) into (3.1) results in a system of ordinary differential equations for $\mathbf{V}$ given by

$$
k^{2} \mathbf{V}^{\prime \prime}-\sigma \mathbf{V}^{\prime}+\mathbf{f}(\mathbf{V})=0,
$$

where prime denotes the differentiation of $\mathbf{V}$ with respect to the independent variable $z$. We want to find $\sigma$ and $k$ so that the last equation has a $2 \pi$-periodic solution for V. We can rewrite system (3.1) as follows:

$$
\frac{\partial}{\partial t}\left(\begin{array}{l}
x_{1} \\
x_{2}
\end{array}\right)=\left(\begin{array}{cc}
\lambda(r) & \mu(r) \\
-\mu(r) & \lambda(r)
\end{array}\right)\left(\begin{array}{l}
x_{1} \\
x_{2}
\end{array}\right)+\frac{\partial^{2}}{\partial x^{2}}\left(\begin{array}{l}
x_{1} \\
x_{2}
\end{array}\right),
$$

where

$$
r^{2}=x_{2}^{2}+x_{2}^{2}, \quad \lambda(r)=\gamma+m r^{2}, \quad \mu(r)=-\left(\omega+n r^{2}\right) .
$$


Now changing the system of equations (3.4) into polar $(r, \theta)$ form, we can write it as follows:

$$
\begin{aligned}
& \frac{\partial r}{\partial t}=r \lambda(r)+r_{x x}-r \theta_{x}^{2}, \\
& \frac{\partial \theta}{\partial t}=\mu(r)+\frac{1}{r^{2}} \frac{\partial}{\partial x}\left(r^{2} \theta_{x}\right) .
\end{aligned}
$$

As we are looking for the travelling wave train solutions of the type (3.2) in polar form, so we substitute

$$
r=\alpha, \quad \theta=\sigma t-k x
$$

into (3.6) to get the necessary and sufficient condition for the existence of travelling wave solution. These conditions are obtained after substitution of (3.7) into (3.6) as

$$
\sigma=\mu(\alpha), \quad k^{2}=\lambda(\alpha)
$$

Considering $\alpha$ as a parameter, the one-parameter family of travelling wave train solutions of (3.4) is given by

$$
x_{1}=\alpha \cos \left[\mu(\alpha) t-x \lambda^{1 / 2}(\alpha)\right], \quad x_{2}=\alpha \sin \left[\mu(\alpha) t-x \lambda^{1 / 2}(\alpha)\right],
$$

with wave speed

$$
c=\frac{\sigma}{k}=\frac{\mu(\alpha)}{\lambda^{1 / 2}(\alpha)}
$$

Such travelling wave trains are of importance, for example, to the target patterns or circular waves generated by the pace-maker nuclei in the BelousovZhabotinski reactions [5, 16, 20].

After finding the travelling wave train solution of the $\lambda-\mu$ system described by (3.4), we now perform the linear stability analysis of the wave train solution. The simplicity of the plane wave solutions in the polar forms (3.6), (3.7), (3.8), and (3.9) gives us the opportunity to do the linear stability analysis. For this linear stability analysis, we consider the perturbations described by

$$
r=\alpha+\rho(x, t), \quad \theta=\sigma t-k x+\phi(x, t),
$$

where $|\rho|,|\phi| \ll 1$. Substituting this relation into (3.6) and then linearizing, we get the following equations in terms of the perturbation variables $\rho$ and $\phi$ as

$$
\begin{aligned}
& \frac{\partial \rho}{\partial t}=\alpha\left[2 m \alpha \rho+2 k \frac{\partial \phi}{\partial x}\right]+\frac{\partial^{2} \rho}{\partial x^{2}} \\
& \frac{\partial \phi}{\partial t}=-2 n \alpha \rho-\frac{2 k}{\alpha} \frac{\partial \rho}{\partial x}+\frac{\partial^{2} \phi}{\partial x^{2}}
\end{aligned}
$$


The conditions satisfied by $k$ and $\sigma$ under which the solutions of (3.12) is to be determined tend to zero as $t$ approaches infinity. As coefficients involved with the system of equations (3.12) are constants, we can assume the solution of the system in the Fourier form

$$
\left(\begin{array}{l}
\rho \\
\phi
\end{array}\right)=\left(\begin{array}{l}
\rho_{0} \\
\phi_{0}
\end{array}\right) \exp (s t+i q x),
$$

where $s$ is the growth rate of perturbation, $q$ is the perturbation wave number, and $\rho_{0}$ and $\phi_{0}$ are constants. The stability of the linearized system demands that $\operatorname{Re}(s)<0$. Substituting (3.13) into (3.12), we get

$$
\left(\begin{array}{cc}
s+q^{2}-2 m \alpha^{2} & -2 i k \alpha q \\
2 n \alpha+2 i k \frac{q}{\alpha} & s+q^{2}
\end{array}\right)\left(\begin{array}{l}
\rho_{0} \\
\phi_{0}
\end{array}\right)=\left(\begin{array}{l}
0 \\
0
\end{array}\right) .
$$

As we are searching for nontrivial solution, then we are not interested in the solution $\rho_{0}, \phi_{0}=0$, so we must have the determinant value of the $2 \times 2$ matrix involved with (3.14) equal to zero, which is a quadratic equation in $s$. If we denote the roots of the quadratic equation by $s_{1}$ and $s_{2}$, then the expressions for the roots are given by

$$
s_{1}, s_{2}=-q^{2}+\alpha^{2} m \pm\left[m^{2} \alpha^{4}+4 k^{2} q^{2}-2 i k n q \alpha^{2}\right]^{1 / 2} .
$$

Depending upon the parameters of the system which are involved in (3.15), the real parts of both $s_{1}$ and $s_{2}$ or of either $s_{1}$ or $s_{2}$ may be positive, and then the plane wave solutions will be linearly unstable. As $s$ and $q$ are perturbation from the plane wave solutions (3.7), then the perturbation wave number $q=0$ leads to the fact that $s_{1}=2 \alpha^{2} m$ and $s_{2}=0$. The later corresponds to the neutral stability while the former implies the stability or instability depending upon the condition satisfied by $m$, and is given by $m<0$ or $m>0$, respectively. For positive perturbation in wave number, that is, $q>0$, the maximum real parts of the roots come from $s_{1}$ and this leads to the necessary and sufficient condition for linear stability, namely, $\operatorname{Re}\left(s_{1}\right)<0$. From (3.15), after some calculations involving complex variable algebra, we find that

$$
\begin{aligned}
\operatorname{Re}\left(s_{1}(q)\right)=-q^{2}+\alpha^{2} m+\frac{1}{\sqrt{2}}[ & \left(m \alpha^{2}\right)^{2}+4 k^{2} q^{2} \\
+ & {\left.\left[\left(\left(m \alpha^{2}\right)^{2}+4 k^{2} q^{2}\right)^{2}+4\left(k n q \alpha^{2}\right)^{2}\right]^{1 / 2}\right]^{1 / 2} }
\end{aligned}
$$

From the above relation, we get

$$
\begin{aligned}
\operatorname{Re}\left(s_{1}(0)\right) & =\alpha^{2} m+\left|\alpha^{2} m\right| \\
{\left[\frac{d \operatorname{Re} s_{1}}{d q^{2}}\right]_{q=0} } & =-1+\frac{4 k^{2}\left(1+n^{2} / m^{2}\right)}{2 \alpha^{2}|m|} .
\end{aligned}
$$


Relation (3.17) states that $\operatorname{Re} s_{1}(0)=2 \alpha^{2} m$ for $m>0$ and $\operatorname{Re} s_{1}(0)=0$ for $m<0$. Thus for small enough $q^{2}, \operatorname{Re} s_{1}(q)<0$ if and only if the last derivative $\left[d \operatorname{Re} s_{1} / d q^{2}\right]_{q=0}<0$. For $m<0$, the relation (3.18) gives the condition as

$$
4 k^{2}\left(1+\frac{n^{2}}{m^{2}}\right)+2 \alpha^{2} m \leq 0
$$

whereas, for $m>0, \operatorname{Re} s_{1}(0)>0$ and consequently the travelling wave train solution of system (3.4) is unstable.

4. Travelling wave in an oscillatory chemical system: linear stability analysis. In this section, we now consider a model chemical reaction as an illustrative example of the general nonlinear system whose different characteristic features we have discussed in the previous section. This model for oscillatory chemical kinetic system was discussed and analyzed by Dreitlein and Somes [4]. Here we analyze its travelling wave solution and its stability. The model is described by the system of nonlinear equations as follows:

$$
\begin{aligned}
& \frac{\partial x_{1}}{\partial t}=E x_{1}+2 x_{2}-x_{1}\left(x_{1}^{2}+x_{2}^{2}\right)+\frac{\partial^{2} x_{1}}{\partial x^{2}}, \\
& \frac{\partial x_{1}}{\partial t}=-2 x_{1}+E x_{2}-x_{2}\left(x_{1}^{2}+x_{2}^{2}\right)+\frac{\partial^{2} x_{2}}{\partial x^{2}} .
\end{aligned}
$$

Comparing this equation with (3.1), we find that $\gamma=E, \omega=-2, m=-1$, and $n=0$. The system of equations (4.1) can be written as a $\lambda-\omega$ model system as follows:

$$
\frac{\partial}{\partial t}\left(\begin{array}{l}
x_{1} \\
x_{2}
\end{array}\right)=\left(\begin{array}{cc}
\lambda(r) & \mu(r) \\
-\mu(r) & \lambda(r)
\end{array}\right)\left(\begin{array}{l}
x_{1} \\
x_{2}
\end{array}\right)+\frac{\partial^{2}}{\partial x^{2}}\left(\begin{array}{l}
x_{1} \\
x_{2}
\end{array}\right)
$$

where

$$
r^{2}=x_{2}^{2}+x_{2}^{2}, \quad \lambda(r)=E-r^{2}, \quad \mu(r)=2 .
$$

Now $r=r_{0}=\sqrt{E}$ is an isolated zero of $\lambda(r)$ and, consequently, $\lambda^{\prime}\left(r_{0}\right)=$ $-2 \sqrt{E}<0, \mu\left(r_{0}\right)=-2 \neq 0$. This leads to the conclusion that the spatially homogeneous system has a limit cycle solution [16]. Changing the variables from $\left(x_{1}, x_{2}\right)$ to the polar variables $(r, \theta)$ and using (3.6), the system of equations (4.1) becomes

$$
\begin{aligned}
& \frac{\partial r}{\partial t}=r\left(E-r^{2}\right)+\frac{\partial^{2} r}{\partial x^{2}}-r\left(\frac{\partial \theta}{\partial x}\right)^{2}, \\
& \frac{\partial \theta}{\partial t}=2+\frac{1}{r^{2}} \frac{\partial}{\partial x}\left(r \frac{\partial \theta}{\partial x}\right) .
\end{aligned}
$$

As $r_{0}=\sqrt{E}>0$ and $\lambda^{\prime}(\sqrt{E})<0$, then the asymptotically stable limit cycle solution of the kinetic system is given by

$$
r=\sqrt{E}, \quad \theta=\theta_{0}+2 t
$$


where $\theta_{0}$ is some arbitrary phase. Next, we look for travelling wave solution of the form (3.3) of the system governed by the system of differential equation (4.1). Substituting $r=\alpha, \theta=\sigma t-k x$ in (4.4), we can obtain the necessary and sufficient conditions for the solution of the above-mentioned type. The conditions are

$$
\sigma=2, \quad k^{2}=E-\alpha^{2} .
$$

The one-parameter family of travelling wave train solutions of (4.4) or, equivalently, for (4.1), is given by

$$
x_{1}=\alpha \cos \left[2 t-x\left(E-\alpha^{2}\right)^{1 / 2}\right], \quad x_{2}=\alpha \sin \left[2 t-x\left(E-\alpha^{2}\right)^{1 / 2}\right],
$$

with $\alpha$ as the convenient parameter. As the parameter $\alpha$ approaches the value $r_{0}=\sqrt{E}$, the wave number of the plane waves tends to zero and this indicates the existence of travelling plane wave train solutions near the limit cycle. The system governed by $(4.1)$ has a steady state at $(0,0)$ which is stable for $E>0$ and unstable for $E<0$. Note that $E=E_{c}=0$ is the bifurcation value of the system. At the critical value $E_{c}=0$, the eigenvalues of the linearized system about the steady state are $\pm 2 i$. This satisfies the requirements of Hopf-bifurcation that we have discussed in Section 2.

Our next task is to investigate the linear stability of the wave train solution that we have discussed for system (3.1) in Section 3. In a similar manner as that we have adopted in the previous section, we will deal with the polar form (4.4) and the perturbations will be of the form

$$
r=\alpha+\rho(x, t), \quad \theta=\sigma t-k x+\phi(x, t),
$$

where $|\rho|,|\phi| \ll 1$. Substituting this relation into (4.4) and linearizing, we get

$$
\begin{aligned}
\frac{\partial \rho}{\partial t} & =\alpha\left[-2 \alpha \rho+2 k \frac{\partial \phi}{\partial x}\right]+\frac{\partial^{2} \rho}{\partial x^{2}}, \\
\frac{\partial \phi}{\partial t} & =-\frac{2 k}{\alpha} \frac{\partial \rho}{\partial x}+\frac{\partial^{2} \phi}{\partial x^{2}} .
\end{aligned}
$$

The coefficients involved in the linearized system (4.9) are all constants and this situation enable us to take the solution of this system in the form

$$
\left(\begin{array}{l}
\rho \\
\phi
\end{array}\right)=\left(\begin{array}{l}
\rho_{0} \\
\phi_{0}
\end{array}\right) \exp (s t+i q x),
$$

where $\rho_{0}, \phi_{0}$ are constants and $q, s$ play the same role as that involved in (3.13). Substituting (4.10) into the system of equations (4.9), we get relations of the form (3.14) as follows:

$$
\left(\begin{array}{cc}
s+q^{2}+2 \alpha^{2} & -2 i k \alpha q \\
2 i k \frac{q}{\alpha} & s+q^{2}
\end{array}\right)\left(\begin{array}{l}
\rho_{0} \\
\phi_{0}
\end{array}\right)=\left(\begin{array}{l}
0 \\
0
\end{array}\right)
$$


As we are interested in the nontrivial solution of system (4.11), then we must have the determinant value of the coefficient matrix involved in (4.11) equal to zero. This determinant value of the above-mentioned matrix equal to zero gives a quadratic equation for the variable $s$. The stability of the linearized system requires that the roots of the quadratic equation in $s$ have negative real parts. If we denote the two roots of the quadratic equation by $s_{1}, s_{2}$, then the expressions for them can be given as follows (using the expression (3.15)):

$$
s_{1}, s_{2}=-q^{2}-\alpha^{2} \pm\left[\alpha^{4}+4 k^{2} q^{2}\right]^{1 / 2} .
$$

From the above relation we get $s_{1}(0), s_{2}(0)=0,-2 \alpha^{2}$. Thus, for vanishingly small perturbation in the wave number, the linearized system exhibits a neutral stability. Now, for small perturbation, both roots $s_{1}$ and $s_{2}$ will be negative if and only if

$$
2 k^{2}-\alpha^{2}<0
$$

This is the condition for stability of the linearized system and it is independent of the parameter $E$ involved with system (4.1).

5. Conclusion. The bifurcation theory plays a significant role in the behaviour of nonlinear systems. The bifurcating behaviour for a nonlinear system is a self-developed phenomena for the deterministic system [10]. The first problem in this paper is the study of an interacting homogeneous population system governed by the nonlinear system of differential equations (2.1). The Hopf-bifurcation analysis of the system leads to an unstable or a stable limit cycle according to whether the bifurcation parameter is negative or positive. The limit cycle solution (2.8) of the nonlinear system (2.1) shows an interesting characteristic that the existence of the limit cycle solution depends upon the sign of the parameter $\gamma$ and $m$ involved with the system. The existence of limit cycle solution of system (2.1) demands $m \gamma<0$. However, when $m=0$, although the conditions for Hopf-bifurcation are satisfied, there are no periodic orbits in the vicinity of the bifurcating point.

The next problem is concerned with the study of travelling wave train solution of the diffusive nonlinear dynamical system and the linear stability criteria of this wave train solution. Equation (3.9) represents the one-parameter family of wave train solution for system (3.1), where $\alpha$ is the arbitrary parameter. If $r=r_{0}$ is an isolated zero of $\lambda(r)$ (given by (3.5)), then the limiting approach $r_{0} \rightarrow \alpha$ gives the small amplitude travelling wave train solution near the limit cycle arising from Hopf-bifurcation. Kopell and Howard [13, 14] showed how to do this in general. Feroe [7] has discussed the difficulties in the stability of travelling wave train solution for excitable FHN waves. However, we are able to 
find this criteria for system (3.1) given by (3.19) without long and complicated calculations due to the simplicity of the travelling wave train [16].

As an illustrative example, we have considered the chemical reaction-diffusion model introduced by Dreitlein and Somes [4]. The travelling wave train solution of the model system (4.1) given by (4.7) is quite similar to the result introduced by Dreitlein and Somes. Condition (4.13) is the linear stability condition of the wave train solution (4.7) and it should be noted that (4.13) does not contain the parameter $E$ of the model system (4.1). The stability condition (4.13) may be derived from (3.19) by substituting the values of $m$ and $n$ for the model system (4.1).

\section{REFERENCES}

[1] S. Banerjee, R. Bhattacharya, and C. G. Chakrabarti, Shift of bifurcation point due to noise induced parameter, Int. J. Math. Math. Sci. 23 (2000), no. 6, 435439.

[2] Y. Chen and A. Y. T. Leung, Bifurcation and Chaos in Engineering, Springer-Verlag, New York, 1998.

[3] D. S. Cohen, J. C. Neu, and R. R. Rosales, Rotating spiral wave solutions of reactiondiffusion equations, SIAM J. Appl. Math. 35 (1978), no. 3, 536-547.

[4] J. Dreitlein and M. L. Somes, A model for oscillatory kinetic systems, J. Theoret. Biol. 46 (1974), 559-572.

[5] M. R. Duffy, N. F. Britton, and J. D. Murray, Spiral wave solutions of practical reaction-diffusion systems, SIAM J. Appl. Math. 39 (1980), no. 1, 8-13.

[6] L. Edelstein-Keshet, Mathematical Models in Biology, The Random House/ Birkhäuser Mathematics Series, Random House, New York, 1988.

[7] J. A. Feroe, Existence and stability of multiple impulse solutions of a nerve equation, SIAM J. Appl. Math. 42 (1982), no. 2, 235-246.

[8] P. Grindrod, The Theory and Applications of Reaction-Diffusion Equations. Patterns and Waves, Oxford Applied Mathematics and Computing Science Series, The Clarendon Press, Oxford University Press, New York, 1996.

[9] B. D. Hassard, N. D. Kazarinoff, and Y. H. Wan, Theory and Applications of Hopf Bifurcation, London Mathematical Society Lecture Note Series, vol. 41, Cambridge University Press, Cambridge, 1981.

[10] L. N. Howard, Nonlinear oscillations, Nonlinear Oscillations in Biology (Proc. Tenth Summer Sem. Appl. Math., Univ. Utah, Salt Lake City, Utah, 1978) (F. C. Hoppensteadt, ed.), Lectures in Appl. Math., vol. 17, American Mathematical Society, Rhode Island, 1979, pp. 1-67.

[11] J. H. Hubbard and B. H. West, Differential Equations: a Dynamical Systems Approach. Higher-Dimensional Systems, Texts in Applied Mathematics, vol. 18, Springer-Verlag, New York, 1995.

[12] _ Differential Equations: a Dynamical Systems Approach. Ordinary Differential Equations, Texts in Applied Mathematics, vol. 5, SpringerVerlag, New York, 1995.

[13] N. Kopell and L. N. Howard, Horizontal bands in the Belousov reaction, Science 180 (1973), 1171-1173.

[14] _ Plane wave solutions to reaction-diffusion equations, Studies in Appl. Math. 52 (1973), 291-328.

[15] Y. Kuramoto and T. Yamada, Pattern formation in oscillatory chemical reactions, Progr. Theoret. Phys. 56 (1976), no. 3, 724-740. 
[16] J. D. Murray, Mathematical Biology, Biomathematics, vol. 19, Springer-Verlag, Berlin, 1993.

[17] A. H. Nayfeh and B. Balachandran, Applied Nonlinear Dynamics, Wiley Series in Nonlinear Science, John Wiley \& Sons, New York, 1995.

[18] L. Perko, Differential Equations and Dynamical Systems, Texts in Applied Mathematics, vol. 7, Springer-Verlag, New York, 1991.

[19] F. Verhulst, Nonlinear Differential Equations and Dynamical Systems, SpringerVerlag, New York, 2000.

[20] A. T. Winfree, Spiral waves of chemical activity, Science 175 (1972), 634-636.

[21] _ _ Rotating chemical reactions, Sci. Amer. 230 (1974), no. 6, 82-95.

Malay Bandyopadhyay: Department of Applied Mathematics, University of Calcutta, Kolkatta 700 009, India

E-mail address: malay_ban@rediffmai 1 .com

Rakhi Bhattacharya: Department of Applied Mathematics, University of Calcutta, Kolkatta 700 009, India

C. G. Chakrabarti: Department of Applied Mathematics, University of Calcutta, Kolkatta 700 009, India

E-mail address: cgc_math@rediffmai 1.com 


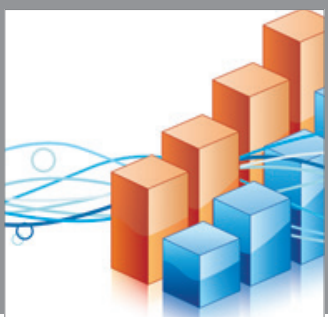

Advances in

Operations Research

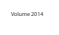

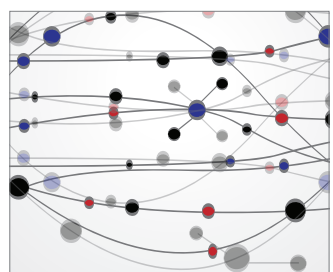

\section{The Scientific} World Journal
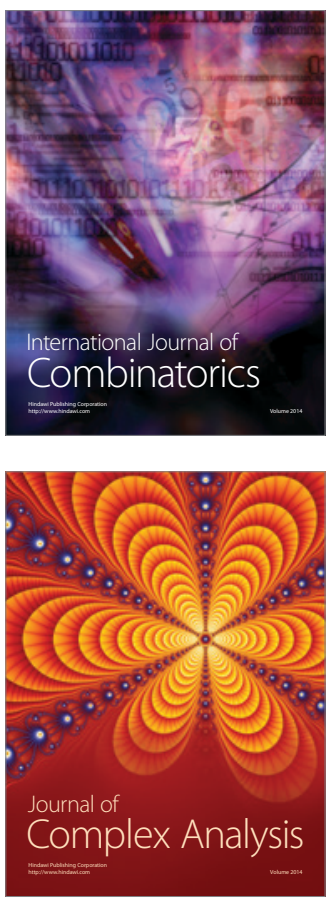

International Journal of

Mathematics and

Mathematical

Sciences
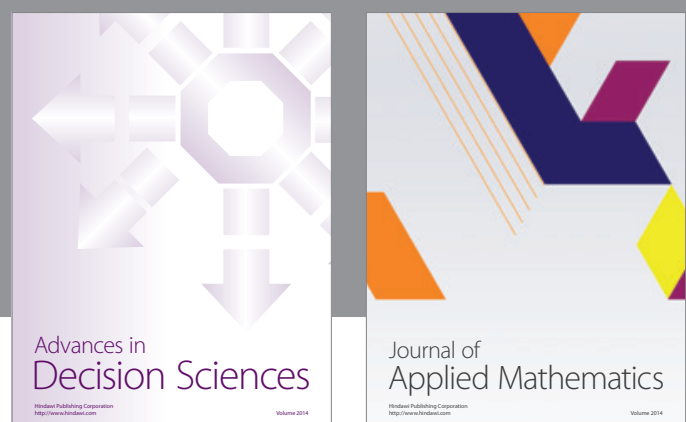

Journal of

Applied Mathematics
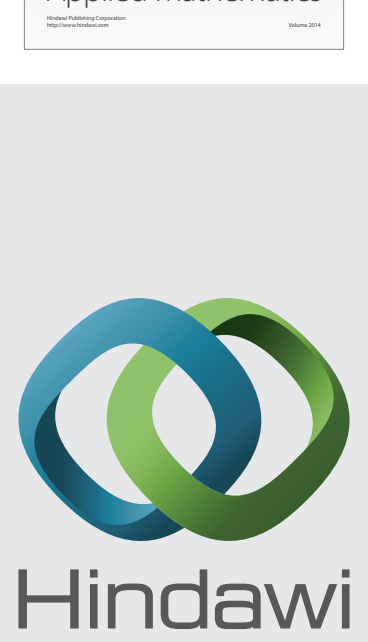

Submit your manuscripts at http://www.hindawi.com
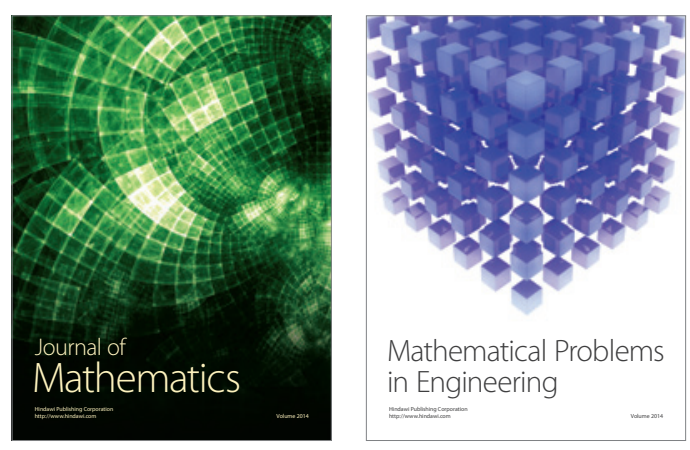

Mathematical Problems in Engineering
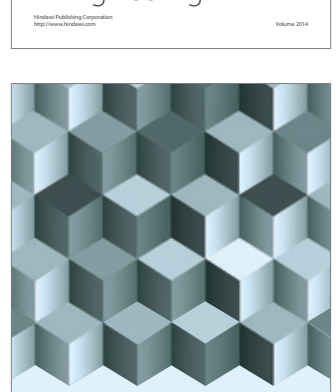

Journal of

Function Spaces
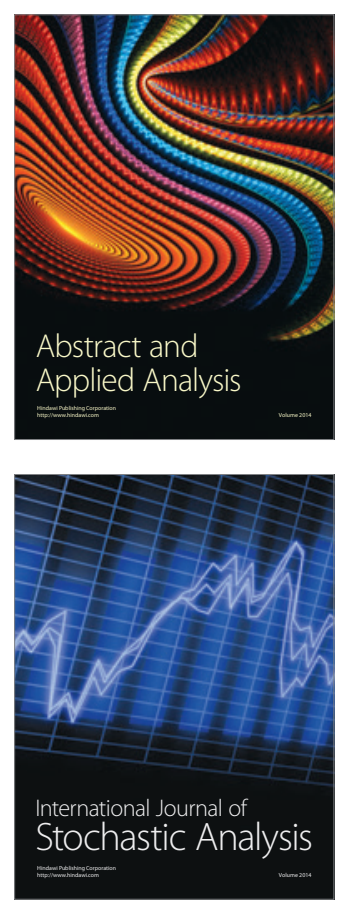

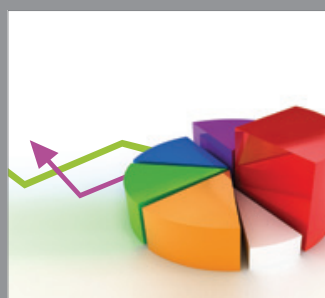

ournal of

Probability and Statistics

Promensencen
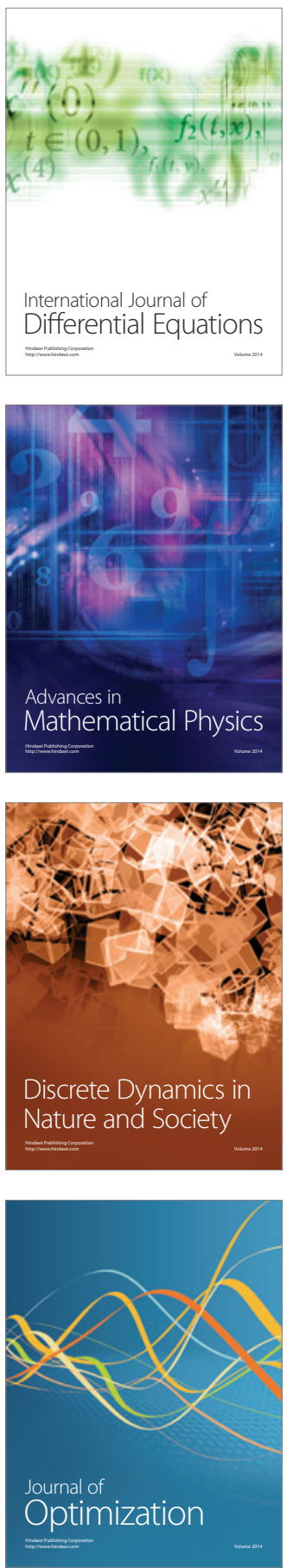